\title{
Comparison of heparinized saline and $0.9 \%$ sodium chloride for maintaining central venous catheter patency in healthy dogs
}

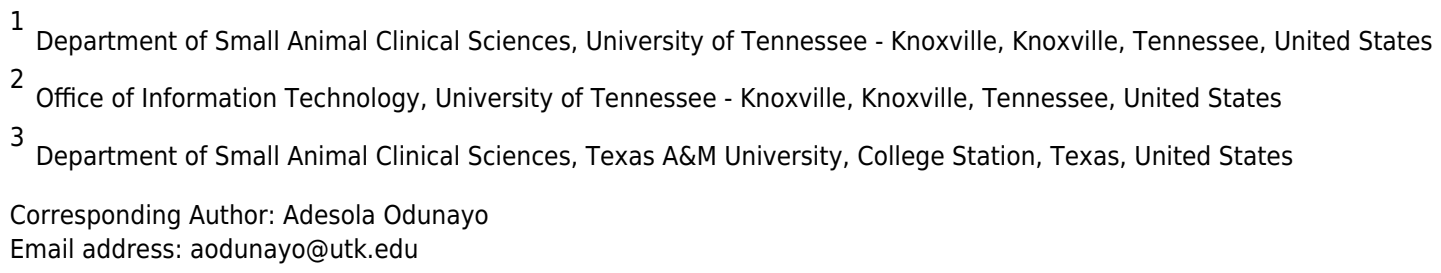

Background. The purpose of this study was to determine whether heparinized saline would be more effective in maintaining the patency of central venous catheters (CVC) in dogs compared to $0.9 \%$ sodium chloride. This was a prospective randomized blinded study conducted at the University Veterinary Teaching Hospital. Methods. Twenty-four healthy purpose bred dogs were randomized into two groups: a treatment and a control group. A CVC was placed in the jugular vein of each dog. Each dog in the treatment group had their CVC flushed with $10 \mathrm{IU} / \mathrm{mL}$ heparinized saline, while dogs in the control group had their CVC flushed with $0.9 \%$ sodium chloride every 6 hours for 72 hours. Immediately prior to flushing, each catheter was evaluated for patency by aspiration of blood. The catheter site was also evaluated for phlebitis, and a rectal temperature was obtained in each dog every six hours. Prothrombin (PT) and activated partial thromboplastin (aPTT) times were evaluated prior to the administration of any flush solution. Results were then compared to values obtained 72 hours later. Results. All CVCs in both groups were patent after 72 hours, which was demonstrated by aspiration of blood and ease of flushing the catheter. Two CVCs in the $0.9 \%$ sodium chloride group had a negative aspiration at hour 12 and 36, respectively. One CVC in the heparinized saline group had a negative aspiration at hour 18. Signs of phlebitis occurred in three dog: two in the $0.9 \%$ sodium chloride group and one in the heparinized saline group. No dog was hyperthermic ( $\left.>103^{\circ} \mathrm{F}\right)$. Two catheters were inadvertently removed by dogs in the heparinized saline group during the study. There were no significant differences in catheter patency, incidence of phlebitis, or incidence of negative aspirations between both groups. APTT and PT values remained within the normal reference range for all dogs in both groups. Ultimately, $0.9 \%$ sodium chloride was as effective as $10 \mathrm{IU} / \mathrm{mL}$ heparinized saline in maintaining the patency of 
central venous catheters for up to 72 hours in healthy dogs. Further evaluation in clinical patients is warranted. 
1 Comparison of Heparinized Saline and $\mathbf{0 . 9 \%}$ Sodium 2 Chloride for Maintaining Central Venous Catheter 3 Patency in Healthy Dogs

4

5

6

7

8 9

42

43

44

45

46

47

48

Julieann Vose ${ }^{1}$, Adesola Odunayo ${ }^{1}$, Joshua Price ${ }^{2}$, Maggie Daves ${ }^{1}$, Julie Christine Schildt ${ }^{1}$, M.

Katherine Tolbert ${ }^{3}$

${ }^{1}$ Department of Small Animal Clinical Sciences, University of Tennessee- Knoxville, Knoxville TN, USA

${ }^{2}$ Office of Information Technology, University of Tennessee-Knoxville, Knoxville, TN, USA

${ }^{3}$ Department of Small Animal Clinical Sciences- Gastrointestinal Laboratory, Texas A\&M University, College Station, TX

Corresponding Author:

Adesola Odunayo ${ }^{1}$

2407 River Dr., Knoxville, TN 37966, USA

Email address: aodunayo@utk.edu 
49

50

51

52

53

54

55

56

57

58

59

60

61

62

63

64

65

66

67

68

69

70

71

72

73

74

75

76

77

78

79

80

81

82

83

84

85

86

87

88

89

90

91

92

93

94

\section{Abstract}

Background. The purpose of this study was to determine whether heparinized saline would be more effective in maintaining the patency of central venous catheters (CVC) in dogs compared to $0.9 \%$ sodium chloride. This was a prospective randomized blinded study conducted at the University Veterinary Teaching Hospital.

Methods. Twenty-four healthy purpose bred dogs were randomized into two groups: a treatment and a control group. A CVC was placed in the jugular vein of each dog. Each dog in the treatment group had their CVC flushed with $10 \mathrm{IU} / \mathrm{mL}$ heparinized saline, while dogs in the control group had their CVC flushed with $0.9 \%$ sodium chloride every 6 hours for 72 hours. Immediately prior to flushing, each catheter was evaluated for patency by aspiration of blood. The catheter site was also evaluated for phlebitis, and a rectal temperature was obtained in each dog every six hours. Prothrombin (PT) and activated partial thromboplastin (aPTT) times were evaluated prior to the administration of any flush solution. Results were then compared to values obtained 72 hours later.

Results. All CVCs in both groups were patent after 72 hours, which was demonstrated by aspiration of blood and ease of flushing the catheter. Two CVCs in the $0.9 \%$ sodium chloride group had a negative aspiration at hour 12 and 36, respectively. One CVC in the heparinized saline group had a negative aspiration at hour 18. Signs of phlebitis occurred in three dog: two in the $0.9 \%$ sodium chloride group and one in the heparinized saline group. No dog was hyperthermic $\left(>103^{\circ} \mathrm{F}\right)$. Two catheters were inadvertently removed by dogs in the heparinized saline group during the study. There were no significant differences in catheter patency, incidence of phlebitis, or incidence of negative aspirations between both groups. aPTT and PT values remained within the normal reference range for all dogs in both groups. Ultimately, $0.9 \%$ sodium chloride was as effective as $10 \mathrm{IU} / \mathrm{mL}$ heparinized saline in maintaining the patency of central venous catheters for up to 72 hours in healthy dogs. Further evaluation in clinical patients is warranted. 
95

96

97

98

99

100

101

102

103

104

105

106

107

108

109

110

111

112

113

114

115

116

117

118

119

120

121

122

123

124

125

126

127

128

129

130

131

132

133

134

135

136

137

138

139

\section{Introduction}

Central venous catheterization was first performed in 1929 and has become a mainstay of modern clinical practice (Smith \& Nolan 2013). They are indispensable devices utilized in both human and veterinary medicine to deliver intermittent medications, continuous intravenous infusions, obtain serial blood draws, provide parental nutrition, aid in hemodynamic monitoring and interventions, and provide access for extracorporeal blood circuits (Elliott et al. 2010; Smith \& Nolan 2013; Tan et al. 2003; Ueda et al. 2013).

Complications associated with indwelling CVCs include phlebitis, occlusion, infection at the catheter site, catheter related blood stream infection, and thrombosis (Parienti et al. 2015). Replacement of CVCs often leads to increased patient discomfort, increased need of sedation, and increased client cost (Cowl et al. 2000; Goode et al. 1991; LeDuc 1997). Although heparin is an effective anticoagulant, its use in preventing thrombosis and maintaining CVC patency has been controversial in human patients (Zhong et al. 2017). While a few human studies showed heparin as more effective at maintaining CVC patency than $0.9 \%$ sodium chloride, in many others a demonstrable advantage of heparin over $0.9 \%$ sodium chloride was not observed (Bradford et al. 2015; Klein et al. 2018; López-Briz et al. 2014; Schallom et al. 2012; Zhong et al. 2017). A recent Cochrane review did not find a demonstrable benefit of heparin over $0.9 \%$ sodium chloride, although the quality of evidence studied was low.(López-Briz et al. 2018)

Complications associated with the use of heparinized saline (HS) include a potentially fatal complication called Heparin-induced Thrombocytopenia (HIT) (McNulty et al. 2005). HIT is an immunological response to heparin in which platelets are activated, causing platelet clumping and clearance from the body (Ahmed et al. 2007; Mian et al. 2017). To the authors' knowledge, HIT has not been documented in dogs. Other complications of heparin use include hypocoagulability, heparin-induced hyperkalemia; allergic reactions; and drug interactions (Mitchell et al. 2009). HS also tends to be more expensive to procure thus increasing the cost of medical care. The process of making HS in veterinary hospitals may also potentially increase the risks of flush contamination. Thus, many human hospitals have discontinued the use of heparinized flushes in hospitalized patients based on consensus statements or guidelines (Ahmed et al. 2007; Barrett \& Lester 1990; FRYKHOLM et al. 2014).. To the authors' current knowledge, there are no studies evaluating the use of HS and $0.9 \%$ sodium chloride in maintaining patency in central venous catheters in dogs.

The purpose of this study was to compare two flush solutions in maintaining patency in central venous catheters placed in dogs compared to $0.9 \%$ sodium chloride. A second objective was to determine if the use of HS caused changes in the aPTT values of healthy dogs. The hypothesisis of this study is that $0.9 \%$ sodium chloride would be equally effective at maintaining central venous catheters as $10 \mathrm{IU} / \mathrm{mL}$ HS in healthy dogs.

\section{Materials \& Methods}

The present study was a prospective, randomized, double-blinded study approved by the Institutional Animal Use and Care Committee at the University of Tennessee (Protocol \#2518). Healthy purpose bred research dogs were utilized in this study. All dogs were deemed healthy based on their physical examination findings, as well as results of a complete blood count, serum 
140 biochemical profile, and urinalysis. The dogs were then randomized into two group: the HS or $141 \quad 0.9 \%$ Sodium Chloride (S) flush group.

142 Butorphanol $(0.4 \mathrm{mg} / \mathrm{kg})$, midazolam $(0.4 \mathrm{mg} / \mathrm{kg})$, ketamine $(2 \mathrm{mg} / \mathrm{kg})$, and dexmedetomidine $(4 \mu \mathrm{g} / \mathrm{kg})$ were administered intravenously through a cephalic vein for sedation. Oxygen supplementation was then provided and physiologic parameters (heart rate, temperature, respiratory rate, electrocardiogram, and blood pressure) were monitored. The dogs were placed in left lateral recumbency, and the area around the right jugular vein was shaved and aseptically prepared. $\mathrm{A} 14 \mathrm{Ga} 20 \mathrm{~cm}$ polyurethane single-lumen $\mathrm{CVC}^{a}$, with a priming volume of $0.35 \mathrm{~mL}$,was placed using the Seldinger technique as described elsewhere (Aspinall 2013). When the CVC could not be successfully placed in the right jugular vein, the left jugular vein was utilized. Two view thoracic radiographs were taken to confirm ideal catheter placement.

Blood was obtained from the CVC immediately after placement and before flushing for assessment of the prothrombin time (PT) and partial thromboplastin time (aPTT). The CVC was secured with sutures, flushed with either HS or S, and a non-adherent dressing ${ }^{b}$ was placed at the insertion site with a bandage applied over the dressing. Needle-free connectors ${ }^{\mathfrak{c}}$ were utilized for all catheters. The dogs were then fitted with an Elizabethan collar after recovery from sedation.

\section{Catheter evaluation}

The CVCs were evaluated by 1 of 3 investigators (JV, MD, or AO). Every dog had its catheter site evaluated for evidence of phlebitis every 6 hours by shifting the neck bandage down and exposing the CVC insertion site. Phlebitis was defined as the presence of any of the following: erythema, tenderness, swelling, unusual discharge, or warmth. A rectal temperature was also obtained from each dog during each evaluation. Hyperthermia was defined as a rectal temperature greater than 103 degrees Fahrenheit.

Catheter patency was evaluated as the ability to aspirate at least $0.5 \mathrm{~mL}$ of blood from the catheter with little resistance. Catheter patency was also assessed by the ability to administer 3 $\mathrm{mL}$ of flush solution with little resistance through the CVC. The flush solution was administered only after the CVC had been evaluated for phlebitis and aspiration of blood had been attempted. All CVCs in the HS and S group were evaluated for phlebitis and patency in the same manner.

\section{Catheter Flushes}

All CVC flushes were performed by 1 of 3 investigators (JV, MD, or AO). The study solutions were prepared by a veterinary technician who was not a part of the study. Each syringe of the study solution was labeled with a predetermined study code to ensure blinded randomization. The dogs in the HS and S groups had their CVCs evaluated and flushed with 3 $\mathrm{mL}$ of $10 \mathrm{IU} / \mathrm{mL}$ HS and $3 \mathrm{~mL}$ of $0.9 \%$ sodium chloride, respectively, every six hours for 72 hours. The catheters were flushed using one quick push of the flush solution, over about 3-5 seconds and the catheters were immediately clamped after the flush was completed. Once enrolled, the subjects continued to receive the same study solution throughout the duration of the study. Catheters were removed upon discovery of non-patency, inadvertent removal by the dog, or at study completion.

\section{Evaluation of Hypocoagulability and Catheter Care}


The CVC insertion site was cleaned with chlorohexidine scrub every 24 hours. The nonadherent dressing and neck wrap were also replaced at this time. The dogs were observed by one of the investigators (JV, MD, or AO) every 3 hours to ensure the CVCs had not been inadvertently removed. Just before the CVC was removed at the end of the study, blood was obtained to evaluate the PT and aPTT for each dog.

\section{Statistical Analysis}

A power analysis calculation was performed prior to the study to determine an appropriate sample size. It was determined that 24 dogs would be a sufficient population to show a difference in catheter occlusion between the HS and S group with a power of .80 of an alpha $<0.05$. Data was analyzed using commercial statistical software. ${ }^{\mathrm{c}}$ A Fisher's exact test was used to analyze whether or not there was a significant overall relationship between whether each dog had an occurrence of phlebitis and the treatment solution they received. A generalized linear mixed model with a binary distribution and logit link function was used to evaluate the occurrence of both phlebitis between the two treatment groups over time. A repeated measure mixed effects ANOVA was used to evaluate the difference in temperature, PT, and PTT values between treatment solutions over time and the solution by time interaction; Holm's p-value adjustment method was applied to post-hoc tests. Shapiro-Wilk normality tests and QQ plots were used to evaluate normality of mixed model ANOVA residuals. Levene's equality of variances test was used to evaluate equality of variances between treatments. All statistical assumptions regarding normality and equality of variances were met.

\section{Results}

Twenty-four purpose breed dogs were enrolled in the study. Twelve dogs were randomly assigned to the $\mathrm{S}$ group, consisting of 4 intact males and 8 intact females while 12 dogs were assigned to the HS group, consisting of 5 intact males and 7 intact females. The mean age of the dogs was 20.33 months (10 months- 69 months). The average weight of the $\mathrm{S}$ and HS groups was $21.25 \mathrm{~kg}(\mathrm{SD} \pm 5.41 \mathrm{~kg})$ and $13.92 \mathrm{~kg} \pm 4.07 \mathrm{~kg}$, respectively. Ten catheters were placed in the right jugular vein, while 2 catheters were placed in the left jugular vein. Both catheters placed in the left jugular vein were accidentally placed in the left jugular due to a communication error during placement. All CVCs were placed during the first attempt without any complications associated with the technical procedure. Two dogs experienced single occasional ventricular premature contractions during catheter placement that resolved without intervention. There were no other complications noted during catheter placement. All catheters terminated at the cranial vena cava on the post placement radiographs.

All CVCs in both groups had catheters that flushed easily at every time point of evaluation. Two of the CVCs in the S group did not aspirate blood back, one at 12 hours and the other at 36 hours, both of which were positive for aspiration of blood at subsequent evaluations. One CVC in the HS group did not aspirate blood back at 18 hours but was positive for aspiration at subsequent evaluations. There was no significant difference in the odds of aspirating blood between the HS and $\mathrm{S}$ treatment groups or over time $(\mathrm{p}=0.70$ and $\mathrm{p}=0.97$, respectively). In the HS group, the study was discontinued after 48 hours in one dog and 54 hours in another dog, due to inadvertent catheter removal by both dogs.

Subjective signs of mild phlebitis were observed in two dogs in the S group at 54 and 72 hours, respectively, and one from the HS group at 48 hours. There was no evidence of ongoing inflammation or patient discomfort, so those CVCs were maintained until the end of the study. 
231 However, there was no significant difference in the odds of phlebitis occurring between groups

232

233

234

235

236

237

238

239

240

241

242

243

244

245

246

247

248

249

250

251

252

253

254

255

256

257

258

259

260

261

262

263

264

265

266

267

268

269

270

271

272

273

274

275

276 or over time ( $\mathrm{p}=0.65$ and $\mathrm{p}=0.68$, respectively).

There was no evidence of hyperthermia recorded in any dog in either the S or HS group. No significant difference in mean temperature between groups was observed $(p=.68)($ Table 1$)$. However, there was a difference in mean temperature over time in both groups $(\mathrm{p}<0.0001)$. The post-hoc test revealed a significant increase in mean temperature of approximately 1.5 degrees between initial observations at time 0 and six hours into observation $(\mathrm{p}=0.0009)$. A significant notable mean increase of approximately 1.8 degrees was observed between initial observations at time 0 and the conclusion of the study after 72 hours $(p<0.0001)$. The maximum mean difference over time of approximately 1.9 degrees occurred between initial observations at time 0 and 24 hours into observation $(\mathrm{p}<0.0001)$.

The PT and aPTT remained within reference ranges for all dogs at all time points (Table 2). There was no significant difference between PT or aPTT values in either treatment group $(\mathrm{p}=0.67$ and $\mathrm{p}=.07$, respectively). Significant pre-test, post-test differences were observed for both PT and aPTT regardless of treatment received ( $\mathrm{p}=0.03$ and $\mathrm{p}=0.003$, respectively). Mean PT values decreased by approximately 0.15 seconds, while aPTT values increased by approximately 1.9 seconds between pre and post measures. There was no evidence of spontaneous or unexplained bleeding in any dog in either group. No CVC had to be removed prematurely because of non-patency, and all CVCs were successfully removed at the end of the study

\section{Discussion}

Despite the overwhelming evidence in human patients showing that $0.9 \%$ sodium chloride is an effective flushing solution to maintain CVC patency (Bradford et al. 2015; Bradford et al. 2016a; Bradford et al. 2016b; Heidari Gorji et al. 2015; LeDuc 1997; Ziyaeifard et al. 2015), heparinized saline is still used in many veterinary facilities (Fleeman 2001). Based on the results of the present study, $0.9 \%$ sodium chloride appears to be a safe alternative to heparin for flushing CVCs and may prove to be more cost effective for facilities, saving both time and money (Ridyard et al. 2017). A previous study evaluating the ideal flush solution for peripheral catheters in dogs had similar findings as the present study (Ueda et al. 2013). A potential reason there was no observable difference between flush solutions used in the present study might be due to the fact that the physical act of flushing (the technique used and the flush volume utilized) plays the most important role in maintaining catheter patency, while the chemical properties of the flush solution itself might play a negligible role (Goossens 2015). Flushing with a turbulent technique (push-pause or pulsatile) enhances the rinsing effect in the catheter and helps prevent catheter malfunction (Goossens 2015). The present study used the quick push technique and its efficacy in preventing catheter malfunction is currently unknown.

Factors associated with the development of intraluminal thrombosis in CVCs include increased length of catheter duration, catheter size, catheter-related sepsis, and infusion of blood products (Park et al. 2014; Timsit et al. 1998). In human patients, symptomatic catheter-related central vein thrombosis is infrequent but asymptomatic catheter-related central vein thrombosis (diagnosed by venographic studies) may be as high as 33\% (Timsit et al. 1998). The use of prophylactic anticoagulants, like heparin, to prevent catheter thrombosis has been studied extensively in critically ill human patients (Akl et al. 2011; Dal Molin et al. 2014; Kirkpatrick et al. 2007; Schallom et al. 2012; Vidal et al. 2014). In some studies, systemic anticoagulation of the patient may help reduce thromboembolic disease. However, there is no strong evidence in human patients that heparin flushes reduce the incidence of intraluminal thrombosis in CVCs. 
277 Indeed, anticoagulant prophylaxis to prevent intraluminal CVC thrombosis is not recommended 278 in human patients (Farge et al. 2013; Schallom et al. 2012; Smith \& Nolan 2013).

279

280

281

282

283

284

285

286

287

288

289

290

291

292

293

294

295

296

297

298

299

300

301

302

303

304

305

306

307

308

309

310

311

312

313

314

315

316

317

318

319

320

321

322

Three catheters in the present study had one episode of inability to withdraw blood during one evaluation point, although all three catheters flushed easily at each of those time points. It is likely that the inability to withdraw blood had to do with the position of the dog's neck or mechanical kinking, which may alter the position of the tip of the CVC and potentially interfere with the ability to withdraw blood (Stephens et al. 1995). However, it is also possible that all three catheters had intraluminal thrombosis, which inhibited blood withdrawal, also known as the "ball valve" effect. The "ball valve" effect is thought to be caused by fibrin obstructing the catheter tip when negative pressure is applied in an attempt to withdraw blood but does not obstruct the flow of flush solution through it (Stephens et al. 1995). However, in human patients, the inability to withdraw blood does not predict the presence of intraluminal thrombosis (Kuter 2004). In one study, $57 \%$ of thrombosed CVCs compared to $27 \%$ of nonthrombosed CVCs failed to allow blood to be drawn (Gould et al. 1993; Kuter 2004). In a venographic study of CVCs that had problems with blood withdrawal in human patients, $58 \%$ had thrombosis, and $42 \%$ did not have thrombosis. They concluded that nonthrombotic mechanical problems commonly prevent blood flow (Kuter 2004; Stephens et al. 1995). Advanced imaging was not used to determine the reason blood was unable to be withdrawn from the three catheters in this study but may be considered in future studies.

Apart from thrombosis, other CVC related complications reported in human patients include catheter occlusion, CVC blood stream infections, air embolism, catheter obstruction, infection of the insertion site, pulmonary thromboembolism, deep vein thrombosis, and mechanical complications (Blaiset et al. 1995; Parienti et al. 2015). The present study was designed to evaluate for local inflammation (phlebitis and evidence of infection at the insertion site) and, to a degree, systemic infection (fever, lethargy, vomiting, anorexia, or change in attitude of the dogs). There was evidence of local inflammation (phlebitis) in this study, all of which happened after 48 hours of catheterization with no protective value of heparinized flushes on local inflammation. There was no evidence of systemic infection in this study, although this might be due to the short duration of the study. In a recent study evaluating complications associated with CVCs in 47 dogs and cats, only one patient developed signs of inflammation (phlebitis) (Reminga et al. 2018). This patient also had an unexplained fever in conjunction with the local signs of inflammation (thickening of the vessel, erythema, and discomfort). In the present study, there were no localized catheter-related infection identified. It is possible that phlebitis is an uncommon complication of CVCs in veterinary patients, although further studies are warranted to better evaluate this.

There was no evidence of hyperthermia in the present study. The study plan was to evaluate the body temperature of the dogs to see if there might be an increased temperature correlating to the development of phlebitis. There was a very low incidence of phlebitis in the present study, and no dog had any evidence of systemic infection. The difference in temperature over time is largely attributed to mild decreases in temperature following sedation and rebound normothermia once the dogs were awake.

Another potential complication of heparinized flush is the development of hypocoagubility and spontaneous hemorrhage after its continued use. In one case report, a human patient experienced postoperative bleeding as a result from overuse of "heparin flushes" and required a second surgery (Passannante \& Macik 1988). Another objective of the study was to set out to determine if dogs were at risk for spontaneous bleeding due to the use of heparinized 
323 flush. There were no statistically significant differences in the change in aPTT (which is 324 primarily affected with the use of heparin) and PT when the HS and S groups were compared.

325 The significant differences between the PT and aPTT over time, regardless of treatment received,

326

327

328

329

330

331

332

333

334

335

336

337

338

339

340

341

342

343

344

345

346

347

348

349

350

351

352

353

354

355

356

357

358

359

360

361

362

363

364

365

366

367

368 is difficult to explain. These differences are not thought to be clinically significant. However, the mean weight of dogs in this study was $17.58 \mathrm{kgs}$, and the catheters were only flushed 4 times a day. Critically ill veterinary patients often have their CVCs flushed frequently during the day due to administration of medications or blood draws from the catheter. Thus, the effect of heparinized flushes on the coagulation times of smaller sized dogs, as well as after more frequent flushes, still needs to be evaluated.

In the present study, two dogs experienced ventricular premature contractions during CVC placement. This was likely because the guide wire was in close association with the right atrium during catheter placement. Both dogs recovered uneventfully, and their arrhythmia resolved without therapeutic intervention, once the guidewire was backed out of the dog. Ventricular arrhythmias are commonly reported arrhythmias during CVC placement in human and veterinary patients and generally tend to be self-limiting, although fatal arrhythmias have been reported in human patients (Nayeemuddin et al. 2013; Reminga et al. 2018; Vesely \& Radiology 2003; Yilmazlar et al. 1997). Other potential causes of ventricular premature contractions in these dogs may include hypoxemia, underlying heart disease or as a result of the sedative administered.

One important limitation of this study is that post-hoc observed power calculations resulted in the determination that this study somewhat lacked power to detect differences between treatments. This is likely because there was limited incidence of catheter dysfunction in both groups. While the study was adequately powered to determine differences over time, there was limited power to detect differences between treatments groups. The post hoc analysis determined that XXX dogs will be sufficient to tell a difference in catheter occlusion between both groups. Another limitation of the study is the fact that the study was done in healthy dogs. Critically ill dogs may have underlying alterations in their coagulation system, making them more susceptible to thrombotic events in their CVCs. However, in many studies performed in critically ill human patients, a protective effect of heparin against catheter thrombosis has not been identified. Additional studies, evaluating the protective effects of heparinized saline, should be performed in critically ill dogs. An additional limitation of the current study is the observation period. A duration of 72 hours was chosen based on the subjective duration of CVCs in our institution. Reminga et al. also reported a median duration of 3 days for critically ill dogs and cats with CVC in their institution (Reminga et al. 2018). It is possible that a protective effect of heparin will be seen if the study was performed over a longer period since Reminga et al. concluded that longer catheter dwell times was a risk factor for indwelling complications. Mechanical obstructions (venous thrombosis, kinking) were the most common cause of CVC failure in Reminga et al.'s study (7/47, 14\%); however, they did not specify how many catheters had thrombosis versus kinking, neither did they specify the flush solution used at their institution. The final limitation of the present study is that the investigators who flushed the catheters in the present study subjectively determined the patency of the CVCs. While this is not an objective way of determining catheter patency, it is the same method utilized in clinical patients, both in human and veterinary medicine. Moreover, the investigators were blinded to treatment.

\section{Conclusions}

Peer) reviewing PDF | (2019:01:34754:2:1:NEW 27 Apr 2019) 
369

370

371

372

373

374

375

376

377

378

379

380

381

382

383

384

385

386

387

388

389

390

391

392

393

394

395

396

397

398

399

400

401

402

403

404

405

406

407

408

409

410

411

412

In conclusion, $0.9 \%$ sodium chloride flushes were found to be as effective as $10 \mathrm{IU} / \mathrm{mL}$ heparinized saline flushes in maintaining the patency of central venous catheters in healthy dogs for up to 72 hours.

\section{Acknowledgements}

The authors would like to thank Amanda Hand, MA, Tamberlyn Moyer, LVMT, VTS (Nutrition); Jimmy Hayes, LVMT; Skyler Bowers, LVMT; and Rachel Feuerstein, LVMT for their expertise and technical support.

\section{Footnotes}

a. Arrow central venous catheters. Teleflex, Morrisville, NC, USA

b. Telfa. Cardinal Health, Dublin, $\mathrm{OH}, \mathrm{USA}$

c. MILA international, Inc, Florence, KY, USA

d. SAS Institute Inc., SAS Campus Drive, Cary, North Carolina 27513, USA

\section{References}

Ahmed I, Majeed A, and Powell R. 2007. Heparin induced thrombocytopenia: diagnosis and management update. Postgraduate Medical Journal 83:575-582. 10.1136/pgmj.2007.059188

Akl EA, Vasireddi SR, Gunukula S, Yosuico V, Barba M, Sperati F, Cook D, and Schunemann H. 2011. Anticoagulation for patients with cancer and central venous catheters. Cochrane Database System Review 4.

Aspinall V, Aspinall, R. . 2013. Clinical Procedures in Small Animal Veterinary Practice Elsevier

Barrett PJ, and Lester RL. 1990. Heparin versus saline flushing solutions in a small community hospital. Hospital Pharmacy Journal 25:115-118.

Blaiset M, Couto C, Evans K, and Smeak D. 1995. Complications of indwelling, silastic central venous access catheters in dogs and cats. Journal of the American Animal Hospital Association 31:379384. 10.5326/15473317-31-5-379

Bradford NK, Edwards RM, and Chan RJ. 2015. Heparin versus $0.9 \%$ sodium chloride intermittent flushing for the prevention of occlusion in long term central venous catheters in infants and children. Cochrane Database of Systematic Reviews. 10.1002/14651858.CD010996.pub2

Bradford NK, Edwards RM, and Chan RJ. 2016a. Heparin versus $0.9 \%$ sodium chloride intermittent flushing for the prevention of occlusion in long term central venous catheters in infants and children: A systematic review. Int J Nurs Stud 59:51-59. 10.1016/j.ijnurstu.2016.02.014

Bradford NK, Edwards RM, and Chan RJ. 2016b. Heparin versus $0.9 \%$ sodium chloride intermittent flushing for the prevention of occlusion in long term central venous catheters in infants and children: A systematic review. Int J Nurs Stud 59:51-59. 10.1016/j.ijnurstu.2016.02.014

Cowl CT, Weinstock JV, Al-Jurf A, Ephgrave K, Murray JA, and Dillon K. 2000. Complications and cost associated with parenteral nutrition delivered to hospitalized patients through either subclavian or peripherally-inserted central catheters. Clinical Nutrition 19:237-243. 10.1054/clnu.2000.0103

Dal Molin A, Allara E, Montani D, Milani S, Frassati C, Cossu S, Tonella S, Brioschi D, and Rasero L. 2014. Flushing the central venous catheter: is heparin necessary. Journal of Vascular Access 15:241248.

Peer] reviewing PDF | (2019:01:34754:2:1:NEW 27 Apr 2019) 
413

414

415

416

417

418

419

420

421

422

423

424

425

426

427

428

429

430

431

432

433

434

435

436

437

438

439

440

441

442

443

444

445

446

447

448

449

450

451

452

453

454

455

456

457

458

Elliott KF, Fleeman LM, and Rand JS. 2010. Using 20-gauge percutaneous peripheral catheters to reliably collect serial 4-mL blood samples from conscious dogs. Aust Vet J 88:215-221. 10.1111/j.17510813.2010.00580.x

Farge D, Debourdeau P, Beckers M, Baglin C, Bauersachs R, Brenner B, Brilhante D, Falanga A, Gerotzafias G, and Haim N. 2013. International clinical practice guidelines for the treatment and prophylaxis of venous thromboembolism in patients with cancer. Journal of Thrombosis and Haemostasis 11:56-70.

Fleeman L. 2001. Intermittent heparinised saline flushes for maintaining indwelling peripheral and central intravenous catheters in diabetic dogs. Australian Veterinary Practice 31:126-131.

FRYKHOLM P, PIKWER A, HAMMARSKJÖLD F, LARSSON AT, LINDGREN S, LINDWALL R, TAXBRO K, ÖBERG F, ACOSTA S, and ÅKESON J. 2014. Clinical guidelines on central venous catheterisation. Acta Anaesthesiologica Scandinavica 58:508-524. doi:10.1111/aas.12295

Goode CJ, Titler M, Rakel B, Ones DS, Kleiber C, Small S, and Triolo PK. 1991. A meta-analysis of effects of heparin flush and saline flush: quality and cost implications. Nursing research and practice 40:324-330.

Goossens GA. 2015. Flushing and locking of venous catheters: available evidence and evidence deficit. Nursing research and practice 2015.

Gould JR, Carloss HW, and Skinner WL. 1993. Groshong catheter-associated subclavian venous thrombosis. The American journal of medicine 95:419-423.

Heidari Gorji MA, Rezaei F, Jafari H, and Yazdani Cherati J. 2015. Comparison of the Effects of Heparin and $0.9 \%$ Sodium Chloride Solutions in Maintenance of Patency of Central Venous Catheters. Anesthesiology and Pain Medicine 5:e22595. 10.5812/aapm.22595

Kirkpatrick A, Rathbun S, Whitsett T, and Raskob G. 2007. Prevention of central venous catheterassociated thrombosis: a meta-analysis. The American journal of medicine 120:901. e901-901. e913.

Klein J, Jepsen A, Patterson A, Reich RR, and Mason TM. 2018. Heparin Versus Normal Saline: Flushing Effectiveness in Managing Central Venous Catheters in Patients Undergoing Blood and Marrow Transplantation. Clinical journal of oncology nursing 22:199-202. 10.1188/18.cjon.199-202

Kuter DJ. 2004. Thrombotic Complications of Central Venous Catheters in Cancer Patients. The Oncologist 9:207-216. 10.1634/theoncologist.9-2-207

LeDuc K. 1997. Efficacy of normal saline solution versus heparin solution for maintaining patency of peripheral intravenous catheters in children. Journal of Emergency Nursing 23:306-309. https://doi.org/10.1016/S0099-1767(97)90216-6

López-Briz E, Garcia VR, Cabello JB, Bort-Marti S, Sanchis RC, and Burls AJCDoSR. 2014. Heparin versus $0.9 \%$ sodium chloride intermittent flushing for prevention of occlusion in central venous catheters in adults.

López-Briz E, Garcia VR, Cabello JB, Bort-Martí S, Sanchis RC, and Burls AJCDoSR. 2018. Heparin versus $0.9 \%$ sodium chloride locking for prevention of occlusion in central venous catheters in adults.

McNulty I, Katz E, and Kim KY. 2005. Thrombocytopenia Following Heparin Flush. Progress in Cardiovascular Nursing 20:143-147. doi:10.1111/j.0889-7204.2005.04693.x

Mian H, Warkentin TE, Sheppard J-AI, MacDonald A, Linkins L-A, Benger A, and Foley R. 2017. Autoimmune HIT due to apheresis catheter heparin flushes for stem cell harvesting before autotransplantation for myeloma. Blood 130:1679-1682. 10.1182/blood-2017-06-788679

Mitchell MD, Anderson BJ, Williams K, and Umscheid CA. 2009. Heparin flushing and other interventions to maintain patency of central venous catheters: a systematic review. Journal of Advanced Nursing 65:2007-2021. doi:10.1111/j.1365-2648.2009.05103.x

PeerJ reviewing PDF | (2019:01:34754:2:1:NEW 27 Apr 2019) 
459

460

461

462

463

464

465

466

467

468

469

470

471

472

473

474

475

476

477

478

479

480

481

482

483

484

485

486

487

488

489

490

491

492

493

494

495

496

497

498

499

500

501

502

503

504

505

Nayeemuddin M, Pherwani AD, and Asquith JR. 2013. Imaging and management of complications of central venous catheters. Clinical Radiology 68:529-544.

https://doi.org/10.1016/j.crad.2012.10.013

Parienti J-J, Mongardon N, Mégarbane B, Mira J-P, Kalfon P, Gros A, Marqué S, Thuong M, Pottier V, Ramakers M, Savary B, Seguin A, Valette X, Terzi N, Sauneuf B, Cattoir V, Mermel LA, and du Cheyron D. 2015. Intravascular Complications of Central Venous Catheterization by Insertion Site. New England Journal of Medicine 373:1220-1229. 10.1056/NEJMoa1500964

Park CK, Paes BA, Nagel K, Chan AK, Murthy P, Thrombosis T, and Group HiN. 2014. Neonatal central venous catheter thrombosis: diagnosis, management and outcome. Blood Coagulation \& Fibrinolysis 25:97-106. 10.1097/MBC.0b013e328364f9b0

Passannante A, and Macik BG. 1988. Case report: the heparin flush syndrome: a cause of iatrogenic hemorrhage. The American journal of the medical sciences 296:71-73.

Reminga CL, Silverstein DC, Drobatz KJ, and Clarke DL. 2018. Evaluation of the placement and maintenance of central venous jugular catheters in critically ill dogs and cats. Journal of Veterinary Emergency and Critical Care 28:232-243. doi:10.1111/vec.12714

Ridyard CH, Plumpton CO, Gilbert RE, and Hughes DA. 2017. Cost-Effectiveness of Pediatric Central Venous Catheters in the UK: A Secondary Publication from the CATCH Clinical Trial. Front Pharmacol 8:644. 10.3389/fphar.2017.00644

Schallom ME, Prentice D, Sona C, Micek ST, and Skrupky LP. 2012. Heparin or $0.9 \%$ sodium chloride to maintain central venous catheter patency: A randomized trial. Critical Care Medicine 40:18201826. 10.1097/CCM.0b013e31824e11b4

Smith RN, and Nolan JP. 2013. Central venous catheters. The BMJ 347:f6570.

Stephens LC, Haire WD, and Kotulak GD. 1995. Are clinical signs accurate indicators of the cause of central venous catheter occlusion? Journal of Parenteral and Enteral Nutrition 19:75-79.

Tan RH, Dart AJ, and Dowling BA. 2003. Catheters: a review of the selection, utilisation and complications of catheters for peripheral venous access. Aust Vet $J$ 81:136-139.

Timsit J-F, Farkas J-C, Boyer J-M, Martin J-B, Misset B, Renaud B, and Carlet J. 1998. Central Vein Catheter-Related Thrombosis in Intensive Care Patients: Incidence, Risks Factors, and Relationship With Catheter-Related Sepsis. Chest 114:207-213. https://doi.org/10.1378/chest.114.1.207

Ueda Y, Odunayo A, and Mann FA. 2013. Comparison of heparinized saline and $0.9 \%$ sodium chloride for maintaining peripheral intravenous catheter patency in dogs. Journal of Veterinary Emergency Critical Care 23:517-522. 10.1111/vec.12093

Vesely TMJJoV, and Radiology I. 2003. Central venous catheter tip position: a continuing controversy. 14:527-534.

Vidal E, Sharathkumar A, Glover J, and Faustino EVS. 2014. Central venous catheter-related thrombosis and thromboprophylaxis in children: a systematic review and meta-analysis. Journal of Thrombosis and Haemostasis 12:1096-1109. 10.1111/jth.12598

Yilmazlar A, Bilgin H, Korfali G, Eren A, and Özkan UJJotRSoM. 1997. Complications of 1303 central venous cannulations. 90:319-321.

Zhong L, Wang H-L, Xu B, Yuan Y, Wang X, Zhang Y-y, Ji L, Pan Z-m, and Hu Z-S. 2017. Normal saline versus heparin for patency of central venous catheters in adult patients - a systematic review and meta-analysis. Critical Care 21:5. 10.1186/s13054-016-1585-x

Ziyaeifard M, Alizadehasl A, Aghdaii N, Sadeghi A, Azarfarin R, Masoumi G, and Golbargian G. 2015. Heparinized and Saline Solutions in the Maintenance of Arterial and Central Venous Catheters After Cardiac Surgery. Anesthesiology and Pain Medicine 5:e28056. 10.5812/aapm28056

PeerJ reviewing PDF | (2019:01:34754:2:1:NEW 27 Apr 2019) 


\section{Table $\mathbf{1}$ (on next page)}

Mean rectal temperature over time in 24 healthy dogs with $0.9 \%$ sodium chloride or heparinized saline flushes

Mean rectal temperature and standard deviation of 24 healthy dogs with $0.9 \%$ sodium chloride $(n=12)$ or heparinized saline $(n=12)$ central venous catheter flushes. Significant differences were observed over time $(P<0.0001)$ but not between treatments. Mean rectal temperatures that do not share a common superscript letter differed significantly $(P<0.05)$ based on Holm's post-hoc analysis. 

Hour
10 IU/mL Heparinized Saline (HS)

\begin{tabular}{lll}
\hline $\mathbf{0}$ & $99.00 \pm 1.52^{\mathrm{D}}$ & $99.00 \pm 1.41^{\mathrm{D}}$ \\
\hline $\mathbf{6}$ & $100.39 \pm 1.08^{\mathrm{ABC}}$ & $100.58 \pm 0.51^{\mathrm{ABC}}$ \\
\hline $\mathbf{1 2}$ & $100.57 \pm 0.91^{\mathrm{BC}}$ & $100.23 \pm 0.48^{\mathrm{BC}}$ \\
\hline $\mathbf{2 4}$ & $100.62 \pm 0.69^{\mathrm{ABC}}$ & $100.53 \pm 0.94^{\mathrm{ABC}}$ \\
\hline $\mathbf{3 0}$ & $100.74 \pm 0.68^{\mathrm{A}}$ & $101.07 \pm 0.77^{\mathrm{A}}$ \\
\hline $\mathbf{3 6}$ & $100.60 \pm 1.05^{\mathrm{ABC}}$ & $100.36 \pm 0.50^{\mathrm{ABC}}$ \\
\hline $\mathbf{4 2}$ & $100.45 \pm 0.80^{\mathrm{BC}}$ & $100.39 \pm 0.86^{\mathrm{BC}}$ \\
\hline $\mathbf{4 8}$ & $100.3 \pm 0.49^{\mathrm{BC}}$ & $100.73 \pm 0.73^{\mathrm{AB}}$ \\
\hline $\mathbf{5 4}$ & $100.58 \pm 0.47^{\mathrm{AB}}$ & $100.13 \pm 0.50^{\mathrm{C}}$ \\
\hline $\mathbf{6 0}$ & $100.15 \pm 0.34^{\mathrm{C}}$ & $99.90 \pm 0.34^{\mathrm{C}}$ \\
\hline $\mathbf{6 6}$ & $100.25 \pm 0.61^{\mathrm{C}}$ & $99.77 \pm 0.35^{\mathrm{CD}}$ \\
\hline $\mathbf{7 2}$ & $100.20 \pm 0.91^{\mathrm{CD}}$ & $100.76 \pm 0.57^{\mathrm{AB}}$ \\
\hline
\end{tabular}

1

2 


\section{Table 2 (on next page)}

Mean baseline (T0) and 72 hour (T72) PT and aPTT values of 24 healthy dogs who either had their central venous catheters flushed with $0.9 \%$ sodium chloride group $(n=12)$ or heparinized saline $(n=12)$.

PT $=$ Prothrombin time, aPTT $=$ Activated Partial Thromboplastin Time $* \mathrm{P}=0.03+\mathrm{P}=0.003 \ddagger$ $P=0.676 \S P=0.069$ 
Mean PT, (S) Mean aPTT, (S)

\begin{tabular}{lcc}
\hline $\mathbf{0 . 9 \%}$ Sodium Chloride T0 & $8.45 \pm 0.57 \mathrm{~s} * \ddagger$ & $15.49 \pm 2.23 \mathrm{~s}+\S$ \\
\hline $\mathbf{0 . 9 \%}$ Sodium Chloride T72 & $8.26 \pm 0.41 \mathrm{~s}^{*}$ & $17.06 \pm 3.87 \mathrm{~s}{ }^{\dagger}$ \\
\hline $\mathbf{1 0 U} / \mathbf{m L}$ Heparinized Saline T0 & $8.64 \pm 1.79 \mathrm{~s} * \ddagger$ & $18.50 \pm 5.66 \mathrm{~s}+\S$ \\
\hline $\mathbf{1 0 U} / \mathbf{m L}$ Heparinized Saline T72 & $8.06 \pm 0.51 \mathrm{~s}^{*}$ & $19.12 \pm 2.57 \mathrm{~s} \dagger$
\end{tabular}

$2 \mathrm{PT}=$ Prothrombin time, aPTT $=$ Activated Partial Thromboplastin Time $* \mathrm{P}=0.03+\mathrm{P}=0.003 \ddagger$

$3 \mathrm{P}=0.676 \S \mathrm{P}=0.069$

4

5 